\title{
A redesigned CRISPR/Cas9 system for marker-free genome editing in Plasmodium falciparum
}

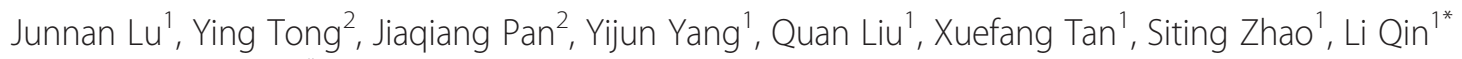
and Xiaoping Chen ${ }^{1 *}$

\begin{abstract}
Background: A highly efficient CRISPR/Cas9-based marker-free genome editing system has been established in Plasmodium falciparum (Pf). However, with the current methods, two drug-selectable markers are needed for episome retention, which may present hurdles for consecutive genome manipulations due to the limited number of available selectable markers. The loading capacity of donor DNA is also unsatisfactory due to the large size of the Cas9 nuclease and sgRNA co-expression system, which limits the size of knock-in DNA fragments. Because of the inefficient end joining (EJ) DNA repair mechanism of Pf, a suicide-rescue approach could be used to address the challenges. Cas9 nuclease and sgRNA were co-expressed from a single plasmid (suicide vector) with one selectable marker, and the donor DNA was ligated into the other plasmid (rescue vector) containing only the ampicillinresistance gene (AmpR) and a ColEl replication origin (ori). Nonetheless, whether this approach can mediate even the regular gene editing in Pf remains unknown. This study aimed to demonstrate the basic gene editing function of this Cas9-mediated suicide-rescue system.

Findings: The suicide and rescue vectors were constructed and co-transfected into Pf3D7. This system worked as expected when used to disrupt the Pfset2 gene and to insert a green fluorescent protein-renilla luciferase (gfp-ruc) fusion gene cassette of 3334 base pairs (bp) into the Pf47 locus, demonstrating that the suicide vector actually induced double-strand breaks (DSBs) and that the rescue vector functioned without maintenance via drug selection.

Conclusions: The adapted marker-free CRISPR/Cas9 system with only a single episome-selectable marker performs well as the current systems for general gene editing which lays a solid foundation for further studies including consecutive gene manipulations and large gene knock-ins.
\end{abstract}

Keywords: Marker-free, CRISPR/Cas9, Plasmodium falciparum

\section{Findings}

\section{Background}

Malaria is still a major public health burden in many countries, and the most lethal malaria parasite is Plasmodium falciparum $(P f)[1,2]$. An understanding of the gene function of $P f$ and the performance of pathogen genetic manipulations is essential for anti-

\footnotetext{
*Correspondence: qin_li@gibh.ac.cn; chen_xiaoping@gibh.ac.cn

'Laboratory of Pathogen Biology, State Key Laboratory of Respiratory Disease, Center for Infection and Immunity, Guangzhou Institutes of Biomedicine and Health (GIBH), Chinese Academy of Sciences, No. 190 Kaiyuan Avenue, Guangzhou Science Park, Guangzhou 510530, Guangdong Province, China

Full list of author information is available at the end of the article
}

malaria drug and malaria vaccine development. The traditional approach to genome modification in $P f$ relies on single- or double-crossover recombination $[3,4]$ and is time-consuming and labor intensive. Moreover, the knock-in of larger gene cassettes without selectable marker integration is impractical using such strategies.

The newly developed genome-editing technology CRISPR/Cas9 has been successfully used in $P f$ and exhibits higher efficiency. Transgenic parasites can be obtained within a typical timeframe of 3-6 weeks, and both marker-integrated and marker-free systems 
are available $[5,6]$. In the system developed by Ghorbal et al. [5], Cas9 nuclease and sgRNA are expressed under the endogenous Hsp86 and U6 promoters and form a complex with targeted DNA strands to induce double-strand breaks (DSBs) with high efficiency. This process results is considerable target gene replacement with the drug-selectable marker-cassette human dihydrofolate reductase (hdhfr). Site-specific mutation without the integration of a selectable marker can also be efficiently achieved by maintaining the CRISPR/Cas9 system and the donor DNA loading plasmids as episomes using two selectable markers, hdhfr and yeast dihydroorotate dehydrogenase $(y D H O D H)$. In another CRISPR/Cas9 system for $P f$ adapted by Wagner et al. [6], T7 RNA polymerase (T7 RNAP) is introduced for sgRNA transcription. As a marker-free genome-editing tool, blasticidin $\mathrm{S}$ deaminase $(b s d)$ and neomycin phosphotransferase (neo) are employed to maintain the plasmids carrying the T7 RNAP transcribe system, CRISPR/Cas9 system and donor DNA. This T7 RNAP-dependent system yields $50-100 \%$ gene knockout frequencies within the usual time frame. The successful application of this powerful tool promises to accelerate both basic and applied $P f$ research.

However, the current CRISPR/Cas9 systems may not be competent for consecutive genome manipulations in $P f$ due to the limited number of available selection markers and potential drug incompatibility. Moreover, the loading capacity for donor DNA is also unsatisfactory; indeed, current systems may not accept larger exogenous DNA fragments for knock-in. As described above, in the system developed by Wagner et al. [6], T7 RNAP is used for sgRNA transcription, and two plasmids requiring two drugs that must be simultaneously administered for episome retention are used. Additionally, the loading capacity for the donor DNA fragment is limited because the donor plasmid must accept the expression cassettes of the T7 RNAP gene and the drug-selectable marker. The system constructed by Ghorbal et al. [5] is relatively compact due to the use of the endogenous U6 promoter to transcribe the sgRNA, but it also involves two plasmids with two markers and allows limited space for the donor DNA. Although marker-free editing can now be achieved, it may be difficult to perform multiple consecutive genome manipulations, and larger gene cassette knock-ins might suffer technical hurdles.

It has been reported that none of the proteins required for the canonical non-homologous end joining (C-NHEJ) pathway have been identified in Pf [7-9]. The efficiency of EJ DNA repair seems extremely low, although an alternative EJ pathway has been found in
Pf $[6,9,10]$. In view of the inefficient EJ DNA repair in $P f$, a suicide-rescue approach might be appropriate to address these challenges. A common feature of the two systems is that Cas9 nuclease and sgRNA are not co-expressed by a single plasmid perhaps because the plasmid may be too large to be manipulated and may impair the transfection efficiency. If Cas9 nuclease and the sgRNA were encoded on the same plasmid along with the drug selection marker, the site-specific breaks could be induced by just one plasmid (a suicide vector). When the suicide vector is cotransfected with an independently established donor plasmid (a rescue vector) containing the homology arms without another selectable marker, the parasites that receive both plasmids may survive and be selected under drug pressure; the other parasites would die due to the drug or inefficient end-joining DNA repair. Because plasmids lacking the selectable marker would be lost rapidly [8], recovering parasites would theoretically suffer DNA breaks and homologous recombination would occur. The suicide vector would be maintained as an episome, and the next round of genome editing could proceed with the same system but with another drug-selectable marker. Because the donor plasmid is specialized, it can support larger sequences for knock-ins into $P f$. The basic gene editing function of this suicide-rescue approach has been proved using ZFN-mediated gene replacement without a selectable phenotype in Pf [11]. However, whether the Cas9-mediated suicide-rescue system can perform regular gene editing in $P f$ remains unknown. The present study aims to confirm the regular ability of the Cas9-mediated suicide-rescue system and to provide a selectable marker saving, easily operated and low-cost marker-free genome editing tool for $P f$ that may be more suitable for consecutive gene manipulations and also be competent for larger gene cassette knock-ins.

\section{Methods}

\section{Plasmid constructs}

pCas9-BSD-sgRNA (pCBS), the plasmid for Cas9 nuclease, $b s d$ and sgRNA co-expression, was generated in two steps. pL6BSD was first constructed by replacing the hdhfr cassette of pL6-eGFP [5] with the bsd cassette from pCC4 [12] at the restriction sites Sac II and Nco I. Then, the $y f c u$-coding sequence of pL6BSD was replaced with the Cas9 nuclease open reading frame (ORF) from pUF1-Cas9 [5] using the restriction sites Xho I and Kpn I.

For both targets, pCBS-Pfset 2 and pCBS-Pf47 were made by replacing the BtgZ I-adaptor with guide DNA sequence as previously described [5]. The oligonucleotides used for Pfset 2 and Pf47 were P9/P10 
(Additional file 1) and P11/P12, respectively. pGCBSPfset 2 was derived from $p C B S-P f s e t 2$ via the addition of a Thosea asigna virus 2A peptide (GFP-2A)-coding sequence that was amplified using P31/P32 from pBSDv2.1-3 (Additional file 2) at the restriction site Xho I upstream of the Cas9 ORF.

To produce the donor plasmid pARM-Pfsets, the left and right homology arms were amplified using P13/P14 and P15/P16, respectively, from the genomic DNA of Plasmodium falciparum strain 3D7. The two arms were ligated into an intact donor DNA via overlap PCR using P13/P16, and they were then connected to the 'Ampicillin-resistance gene-ColE1 replication origin' ('AmpR-ColE1 ori') fragment, which was amplified using P17/P18 from the commercial Tvector. To detect homologous recombination (HR), a 48-bp DNA fragment was introduced between the arms using P14 and P15. The other donor plasmid, pARM-GFP/RUCki, was constructed by ligating the 'AmpR-ColE1 ori' fragment with the gfp-ruc fusion cassette flanked by the homology arms. The left arm, right arm and $P f$ elongation factor 1-alpha (Pfef1 $\alpha$; [PlasmoDB: PF13_0304]) 5' untranslated region (UTR) were amplified using P19/P20, P21/P22, and P23/P24 from the genomic DNA of 3D7. The gfp ORF, renilla luciferase ORF and Plasmodium berghei dihydrofolate reductase (Pbdhfr; [PlasmoDB: PBANKA_0719300]) terminator (PbDT) were amplified from pLN-GFP (Additional file 2), pHBRIH and the genomic DNA of P. berghei ANKA using P25/P26, P27/P28, and P29/ $\mathrm{P} 30$, respectively. All cloning reactions used the ClonExpress II One-Step Cloning Kit (Vazyme) and XL10-competent cells (Vazyme).

\section{Parasite culture and transfections}

Pf strain 3D7 parasites were routinely cultured in fresh human red blood cells under $5 \% \mathrm{O}_{2}$ and $5 \% \mathrm{CO}_{2}$ in RPMI-1640 media supplemented with $5 \mathrm{~g} / \mathrm{L}$ Albumax I (Thermo Fisher Scientific), $2 \mathrm{~g} / \mathrm{L} \mathrm{NaHCO}_{3}, 25 \mathrm{mM}$ HEPES, $1 \mathrm{mM}$ hypoxanthine and $50 \mathrm{mg} / \mathrm{L}$ gentamicin. The transfections used $\sim 100 \mu \mathrm{g}$ of each plasmid and were performed by the spontaneous DNA uptake method [13]. The transfectants were obtained by selection with $5.0 \mu \mathrm{g} / \mathrm{mL}$ blasticidin $\mathrm{S}$ (Thermo Fisher Scientific).

\section{Whole-cell PCR analysis}

Mutant parasites were verified by PCR using PrimeSTAR GXL DNA Polymerase (Takara Bio, Inc.) according to the manufacturer's instructions. The parasites used as PCR templates were prepared as previously described [14], and cultures at $0.1-10 \%$ parasitaemia and $2 \%$ hematocrit were centrifuged at $350 \times g$ for
$3 \mathrm{~min}$. The pellets were flash-frozen in liquid nitrogen and then thawed, and $1 \mu \mathrm{L}$ of the lysed cells was quickly added to $50 \mu \mathrm{L}$ of the PCR reaction solution on ice to yield a final concentration of $1 \times$ PrimeSTAR GXL Buffer. The primers used to detect the Pfset2 disruption were $\mathrm{P} 1 / \mathrm{P} 2, \mathrm{P} 3 / \mathrm{P} 4$ and $\mathrm{P} 1 / \mathrm{P} 4$. The primers used to detect the $g f p$-ruc fusion cassette knock-in were $\mathrm{P} 5 / \mathrm{P} 6, \mathrm{P} 7 / \mathrm{P} 8$ and $\mathrm{P} 5 / \mathrm{P} 8$. The $\mathrm{PCR}$ products yielded by $\mathrm{P} 1 / \mathrm{P} 4$ and $\mathrm{P} 5 / \mathrm{P} 8$ were purified using an agarose gel extraction kit and sequenced using the same primers.

\section{Fluorescence-activated cell sorting (FACS) of GFP-positive parasites}

The parasite cultures were centrifuged at $350 \times g$ for 3 min, washed, and resuspended with phosphatebuffered saline to a concentration of $1.0 \times 10^{6}$ cells $/ \mathrm{mL}$. The GFP-positive parasites were sorted with a FACSAria $^{\text {ru }}$ II flow cytometer and then used for whole-cell PCR analysis as described above.

Live cell fluorescence microscopy/laser confocal microscopy The parasite cultures were incubated for 10 min with RPMI medium containing $2 \mu \mathrm{g} / \mathrm{mL}$ Hoechst 33,342 (Sigma) at $37{ }^{\circ} \mathrm{C}$ and then washed twice with PBS and applied to a $3.5-\mathrm{cm}$ culture dish (Nunc). Imaging was conducted immediately at room temperature using a Nikon Ti-S inverted microscope or a Zeiss 710 NLO laser confocal microscope.

\section{Luciferase assay}

Renilla luciferase expression was confirmed using the Renilla Luciferase Assay System (Promega). The parasite samples were prepared by centrifugation of the cultures at $\sim 5 \%$ parasitaemia and $2 \%$ hematocrit. Next, $15-\mu \mathrm{L}$ pellets were either used immediately or stored at $-80^{\circ} \mathrm{C}$.

\section{Results and discussion}

We first redesigned and successfully constructed the basic suicide vector pCas9-BSD-sgRNA (pCBS) consisting of the expression cassettes for the sgRNA, Cas9 nuclease and blasticidin $\mathrm{S}$ deaminase. These genes were driven by endogenous promoters. To test the function of this suicide-rescue system, Pfset 2 was chosen as a target because it has been demonstrated to be a nonessential gene for in vitro blood-stage survival of $P f$ [15]. The suicide vector pGFP-CBS-Pfset 2 with a $g f p$ reporter gene was derived from pCBS-Pfset 2 . The donor DNA template consisting of homology arms flanking the Avi-tag was provided by a rescue plasmid, pARM-SET2ko, without a drug-selectable marker for Pf (Fig. 1a). pGFP-CBS-Pfset2 and pARM-SET2ko were co-transfected, and blasticidin $\mathrm{S}$ selection was 


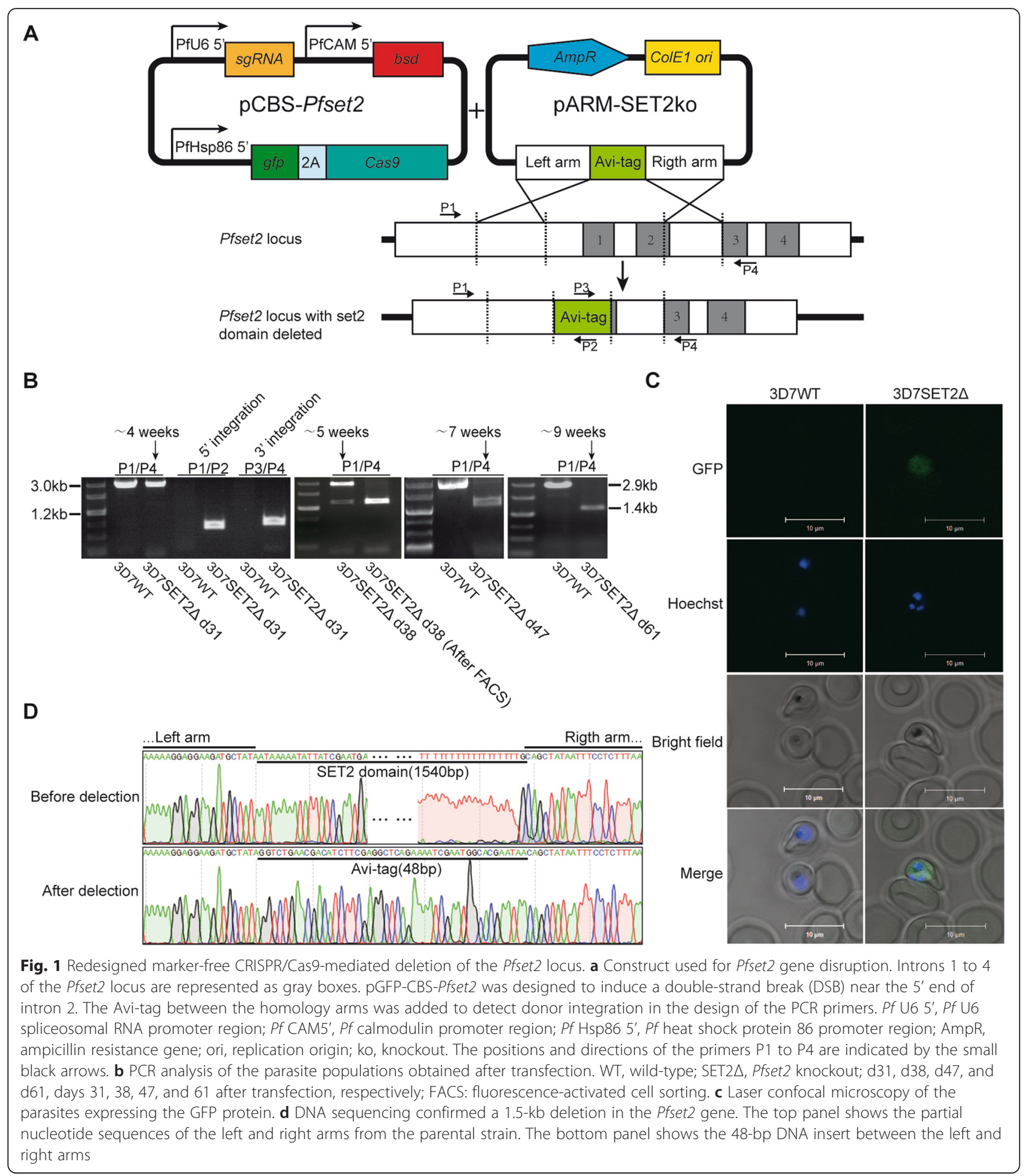

applied. The drug-resistant parasites were collected approximately 4 weeks after transfection, and PCR results confirmed integration of the Avi-tag. However, the truncated locus was nearly undetectable (Fig. 1b). The proportion of edited parasites exhibited an increasing upward tendency as indicated by the subsequent PCR detection (Fig. 1b). One week later, the truncated locus became highly visible (Fig. 1b), and GFP-positive parasites were observed by confocal microscopy (Fig. 1c). The GFP-positive parasites obtained 
by FACS were determined to be edited; no wild-type (WT) loci were detected (Fig. 1b). Additionally, sequencing results indicated the expected editing events (Fig. 1d). The PCR results at day 47 revealed that the mutant parasites became dominant, and the WT locus was nearly undetectable after day 61 (Fig. 1b). Together, these results indicate that the suicide vector actually induced DSBs and that the rescue plasmid also performed well, although it was transiently supplied. Thus, a marker-free genome editing system with only one selectable marker was successfully created. This experiment demonstrated once again that the EJ mechanism of $P f$ is very inefficient.

Currently, the following five drug-selectable markers are available for $P f$ : hdhfr [16], bsd [17], neo [17], $y D H O D H$ [18], and puromycin- $\mathrm{N}$-acetyltransferase $(P A C)$ [19]. Theoretically, at least five rounds of consecutive manipulations could be supported by the suicide-rescue system with each requiring one drug for selection and without consideration for the drug compatibility. Previous systems [5, 6] might support up to 4 rounds of editing, but the Cas9 nucleaseexpressing episome had to be stably maintained beyond the first round of transfection, which might lead to exogenous plasmid integration into the parasite genome.

The suicide-rescue editing system has also been used to mediate the insertion of a $g f p$-ruc fusion gene cassette (a 3337-bp DNA fragment) into the Pf47 locus of $P f$ [20] (Fig. 2a). Live parasites were obtained approximately 4 weeks after transfection. Luciferase assay and fluorescence microscopy results indicated that both genes were functionally expressed (Fig. 2c, d). PCR and sequencing confirmed the insertion of the fusion gene cassette. The WT locus was still detectable at day 51 but was undetectable approximately 12 days later (Fig. 2b). A parallel transfection experiment yielded similar results, and the WT locus was also undetectable by PCR at day 60 . These results illustrate that the redesigned system was capable of mediating the knock-in of larger exogenous gene cassettes and that the extended size of the donor plasmid did not obviously impair the efficiency of cotransfection. Exogenous gene knock-in and endogenous add-back are typically performed in studies of $P f$, but the donor DNA loading capacities of previous systems might limit the sizes of inserted fragments to 10 kilobases $(\mathrm{kb})$ for the donor DNA loading plasmids have exceeded $9.0 \mathrm{~kb}$ [5] or even $11.0 \mathrm{~kb}$ [6]. The rescue plasmid used in this study contains only a 2.1$\mathrm{kb}$ 'AmpR-ori' backbone and thus is capable of providing $7.0 \mathrm{~kb}$ or $9 \mathrm{~kb}$ of extra capacity.

The editing efficiency was not directly assayed in this study. Obviously, the proportion of the parasite population that exhibited editing was dependent on the drug selection time as was observed in both the knock-out and knock-in experiments. According to the previous report, high gene-disruption frequencies $(\geq 50-100 \%)$ can be achieved within the usual timeframe (4-6 weeks) using marker-free CRISPR/Cas9 editing [6]. In our study, the edited population became dominant $(\geq 50 \%)$ in approximately 6-7 weeks, as demonstrated by PCR analysis, and this timeframe is similar to the typical timeframe. Thus it can be seen that the regular drug selection time is not always sufficient for obtaining highly pure edited population ( $100 \%$ editing efficiency) in both previous and redesigned system. In order to avoid the possible plasmid integration, limit dilution and culture without drug pressure should be employed when the gene modified parasites are detectable within the usual timeframe. This process usual needs nearly a month of time for obtaining single-clone parasites with considerable amount for genetic analysis [21] and for next round gene editing. However, this strategy is time consuming and labor intensive because multi-round limited dilution would be needed in consecutive gene manipulation. An alternative method is a compact gene editing process without interruption with limited dilution following the last round manipulation, but the prerequisite is that the highly pure edited population has been generated in the previous gene manipulation. Thus in our study the drug selection time was extended to confirm whether the highly pure edited population could be obtained and how long it would take. In both experiments the time required for the total elimination of the WT parasites (i.e. below the level detectable by PCR) was approximately 9 weeks. This strategy shows no advantages in time consumption compared with limited dilution dependent approach, unless the selection time could be reduced to the usual timeframe. It can be speculated that this extended elimination time was attributable to spontaneous drug resistance in the WT parasite. The GFP-positive parasites obtained by FACS in the knock-out test were part of a highly pure mutant population, which indicated that the 2.9-kb untruncated locus was amplified from spontaneously occurring blasticidinresistant parasites. It has been reported that blasticidin pressure results in resistant transport mutant selection in the FCB strain of $P f$ [22], and 3D7 may also acquire blasticidin resistance through mutation. The time required to reach $100 \%$ edited parasites might be reduced by appropriately increasing the blasticidin concentration.

In conclusion, a suicide-rescue-based marker-free CRISPR/Cas9 system was developed and confirmed 


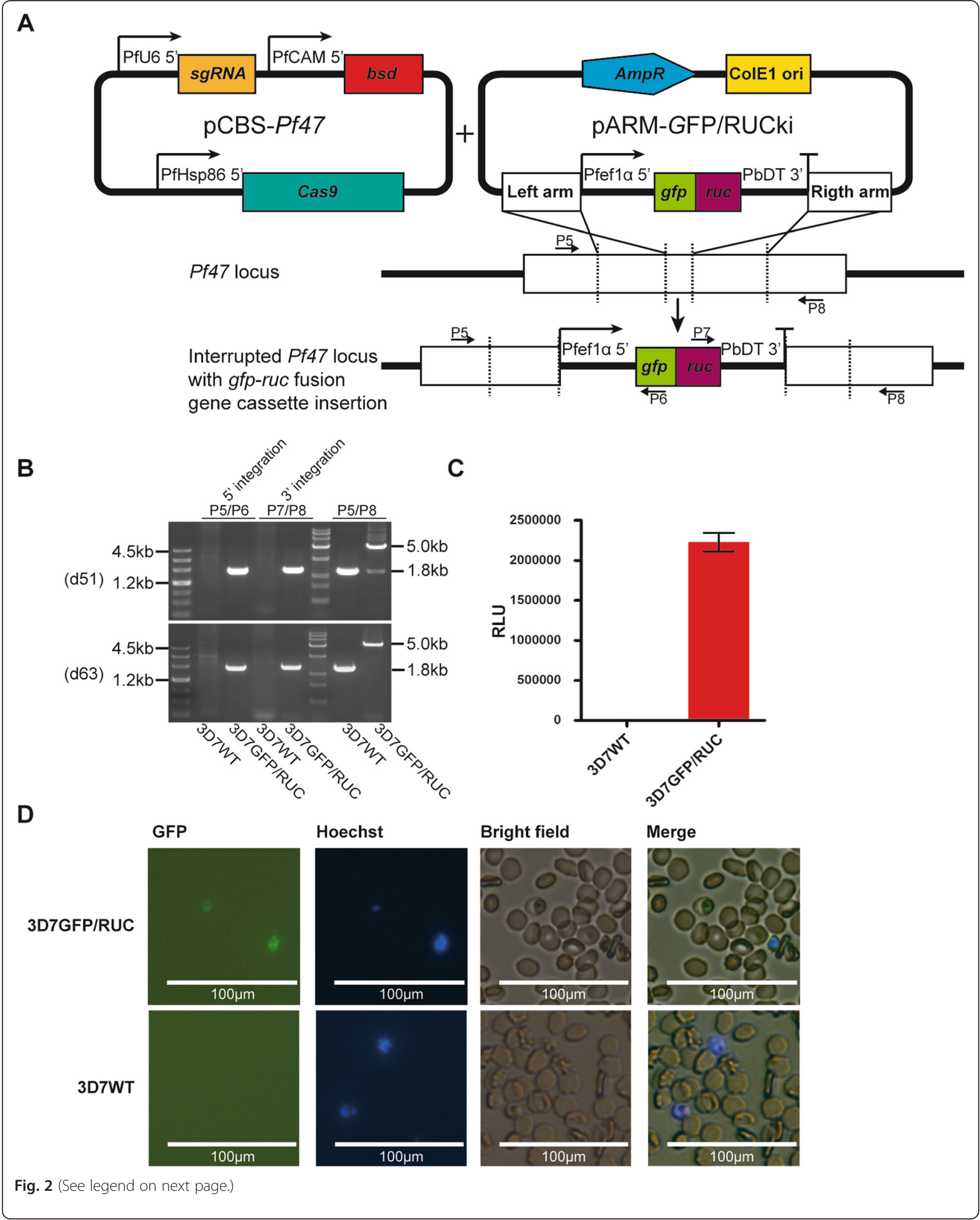


(See figure on previous page.)

Fig. 2 Redesigned marker-free CRISPR/Cas9-mediated insertion of the gfp-ruc fusion gene cassette at the Pf47 locus. a Construct used for gfp-ruc fusion gene cassette insertion into the Pf47 locus. PCBS-Pf47 was designed to target the Pf47 ORF at its $5^{\prime}$ terminal-region, $\sim 150 \mathrm{bp}$ from the start codon. PARM-GFP/RUCki provides a donor fragment with the gfp-ruc fusion cassette flanked by homology arms. The positions and directions of primers P5 to P8 are indicated by small black arrows. Pf EF1a 5', Pf elongation factor 1-alpha promoter region; PbDT 3', Pb dihydrofolate reductase terminator; ki, knock-in. (b) PCR analysis of the parasite populations obtained after transfection. d51 and d63, days 51 and 63 after transfection, respectively. (c) Luciferase assay confirming the functional expression of the integrated ruc gene in the parasites obtained after transfection. The negative control was 3D7 WT. The luciferase assays were performed in triplicate, and the standard deviations are indicated by vertical bars. (d) Live cell fluorescence microscopy of the parasites expressing the GFP protein. The negative control was 3D7 WT

to be competent for general gene manipulations in Pf. This system requires fewer selectable markers and exhibits potential for large gene cassette knockins. This tool may be a useful alternative to Pf genome editing. However, further experiments are needed to confirm whether this system could efficiently mediate consecutive gene manipulations and large gene cassette knock-ins. Although a CRISPR/ Cas9 system has been established in Pf, we call for additional studies to increase the efficiency and versatility of this system.

\section{Additional files}

Additional file 1: PCR primers used in this study. (PDF $35 \mathrm{~kb}$ )

Additional file 2: sequences of pBSDv2.1-3 and pLN-GFP. (PDF $60 \mathrm{~kb}$ )

\section{Abbreviations \\ 2A: Thosea asigna virus 2A peptide; bsd: blasticidin S deaminase; C-NHEJ: canonical non-homologous end joining; DSBs: double-strand breaks; EJ: end joining; FACS: fluorescence-activated cell sorting; gfp-ruc: green fluorescent protein-renilla luciferase; hdhfr: human dihydrofolate reductase; HR: homologous recombination; neo: neomycin phosphotransferase; PAC: puromycin-N-acetyltransferase; Pb: Plasmodium berghei; Pf: Plasmodium falciparum; T7 RNAP: T7 RNA polymerase; yDHODH: yeast dihydroorotate dehydrogenase; $y f c u$ : yeast cytosine deaminase and uridyl phosphoribosyl transferase.}

\section{Competing interests}

The authors declare that they have no competing interests.

\section{Authors' contributions}

$J L$ participated in the experimental design and performed the experiments with contributions from YT, JP and YY. QL, XT and SZ helped with the experimental setup and provided reagents. XC and LiQ supervised all aspects of this study, including the study design, execution, data interpretation and manuscript preparation. All authors have read and approved the final manuscript.

\section{Acknowledgments}

This work was supported by the National Natural Science Foundation of China (No. 31570925, No. 81372451) and the Guangzhou Municipal Funds of Science and Technology (No. 201300000098). We thank Jose-Juan LopezRubio and Mehdi Ghorbal for kindly providing the pUF1-Cas9 and pL6-eGFP plasmids. We thank Xu Zhang at Jilin University for providing plasmid pHBRIH and for technical assistance. We extend special thanks to Lubin Jiang and his group at the Institute Pasteur of Shanghai for help with the $P$. falciparum transfection experiments. We are also grateful to Yi Zheng and Hongwen Pang at the Instrument Center of GIBH for assistance with the use of the instruments.

\section{Author details}

'Laboratory of Pathogen Biology, State Key Laboratory of Respiratory Disease, Center for Infection and Immunity, Guangzhou Institutes of Biomedicine and Health (GIBH), Chinese Academy of Sciences, No. 190 Kaiyuan Avenue, Guangzhou Science Park, Guangzhou 510530, Guangdong Province, China. ${ }^{2}$ CAS Lamvac Biotech Co., Ltd, No. 3 Lanyue Road, Guangzhou Science Park, Guangzhou 510530, Guangdong Province, China.

Received: 16 February 2016 Accepted: 1 April 2016

Published online: 11 April 2016

\section{References}

1. Sharma D, Lather M, Mallick PK, Adak T, Dang AS, Valecha N, et al. Polymorphism in drug resistance genes dihydrofolate reductase and dihydropteroate synthase in Plasmodium falciparum in some states of India. Parasites Vector. 2015;8:471.

2. Mancini MV, Spaccapelo R, Damiani C, Accoti A, Tallarita M, Petraglia E, et al. Paratransgenesis to control malaria vectors: a semi-field pilot study. Parasites Vector. 2016;9(1):140.

3. Wu YM, Kirkman LA, Wellems TE. Transformation of Plasmodium falciparum malaria parasites by homologous integration of plasmids that confer resistance to pyrimethamine. Proc Natl Acad Sci USA. 1996;93(3):1130-4.

4. Duraisingh MT, Triglia T, Cowman AF. Negative selection of Plasmodium falciparum reveals targeted gene deletion by double crossover recombination. Int J Parasitol. 2002;32(1):81-9.

5. Ghorbal M, Gorman M, Macpherson CR, Martins RM, Scherf A, Lopez-Rubio JJ. Genome editing in the human malaria parasite Plasmodium falciparum using the CRISPR-Cas9 system. Nat Biotechnol. 2014;32(8):819-21.

6. $\quad$ Wagner JC, Platt RJ, Goldfless SJ, Zhang F, Niles JC. Efficient CRISPR-Cas9mediated genome editing in Plasmodium falciparum. Nat Methods. 2014; 11(9):915-8

7. Gardner MJ, Hall N, Fung E, White O, Berriman M, Hyman RW, et al. Genome sequence of the human malaria parasite Plasmodium falciparum. Nature. 2002;419(6906):498-511.

8. Aravind L, lyer LM, Wellems TE, Miller LH. Plasmodium biology: genomic gleanings. Cell. 2003;115(7):771-85.

9. Kirkman LA, Lawrence EA, Deitsch KW. Malaria parasites utilize both homologous recombination and alternative end joining pathways to maintain genome integrity. Nucleic Acids Res. 2014;42(1):370-9.

10. Zhang C, Xiao B, Jiang YY, Zhao YH, Li ZK, Gao H, et al. Efficient editing of malaria parasite genome using the CRISPR/Cas9 system. Mbio. 2014;5(4): e01414-14.

11. Straimer J, Lee MCS, Lee AH, Zeitler B, Williams AE, Pearl JR, et al. Site-specific genome editing in Plasmodium falciparum using engineered zinc-finger nucleases. Nat Methods. 2012;9(10):993.

12. Maier AG, Braks JAM, Waters AP, Cowman AF. Negative selection using yeast cytosine deaminase/uracil phosphoribosyl transferase in Plasmodium falciparum for targeted gene deletion by double crossover recombination. Mol Biochem Parasit. 2006;150(1):118-21.

13. Deitsch KW, Driskill CL, Wellems TE. Transformation of malaria parasites by the spontaneous uptake and expression of DNA from human erythrocytes. Nucleic Acids Res. 2001;29(3):850-3.

14. Caro F, Miller MG, DeRisi JL. Plate-based transfection and culturing technique for genetic manipulation of Plasmodium falciparum. Malar J. 2012;11:22.

15. Jiang LB, Mu JB, Zhang QF, Ni T, Srinivasan P, Rayavara K, et al. PfSETvs methylation of histone H3K36 represses virulence genes in Plasmodium falciparum. Nature. 2013;499(7457):223. 
16. Fidock DA, Wellems TE. Transformation with human dihydrofolate reductase renders malaria parasites insensitive to WR99210 but does not affect the intrinsic activity of proguanil. Proc Natl Acad Sci USA. 1997;94(20):10931-6.

17. Ben Mamoun C, Gluzman IY, Goyard S, Beverley SM, Goldberg DE. A set of independent selectable markers for transfection of the human malaria parasite Plasmodium falciparum. Proc Natl Acad Sci USA. 1999;96(19):10944-4.

18. Ganesan SM, Morrisey JM, Ke HJ, Painter HJ, Laroiya K, Phillips MA, et al. Yeast dihydroorotate dehydrogenase as a new selectable marker for Plasmodium falciparum transfection. Mol Biochem Parasit. 2011;177(1):29-34.

19. de Koning-Ward TF, Waters AP, Crabb BS. Puromycin-N-acetyltransferase as a selectable marker for use in Plasmodium falciparum. Mol Biochem Parasit. 2001;117(2):155-60.

20. Talman AM, Blagborough AM, Sinden RE. A Plasmodium falciparum strain expressing GFP throughout the Parasite's life-cycle. PloS One. 2010;5(2): e9156.

21. Doolan DL. Malaria methods and protocols. Totowa, N.J.: Humana Press; 2002.

22. Hill DA, Pillai AD, Nawaz F, Hayton K, Doan L, Lisk G, et al. A blasticidin S-resistant Plasmodium falciparum mutant with a defective plasmodial surface anion channel. Proc Natl Acad Sci USA. 2007;104(3):1063-8.

Submit your next manuscript to BioMed Central and we will help you at every step:

- We accept pre-submission inquiries

- Our selector tool helps you to find the most relevant journal

- We provide round the clock customer support

- Convenient online submission

- Thorough peer review

- Inclusion in PubMed and all major indexing services

- Maximum visibility for your research

Submit your manuscript at www.biomedcentral.com/submit
Biomed Central 